\title{
Peripartum cardiomyopathy: challenges and solutions
}

\author{
Angela Beatrice Scardovi \\ Renata De Maria ${ }^{2}$ \\ 'Division of Cardiology, S. Spirito \\ Hospital, Rome, ${ }^{2} \mathrm{CNR}$ Clinical \\ Physiology Institute, ASST-Great \\ Metropolitan Hospital Niguarda, \\ Milan, Italy
}

This article was published in the following Dove Press journal:

Research Reports in Clinical Cardiology

16 June 2017

Number of times this article has been viewed

\begin{abstract}
Peripartum cardiomyopathy (PPCM) is a rare heart disease which affects previously healthy women toward the end of pregnancy or in the months following delivery. The incidence of the disease shows wide regional variations and has been rising, probably due to greater awareness or socioeconomic changes. The etiology and pathogenesis of PPCM are multifactorial and poorly understood. Several hypotheses have been proposed over the years including myocarditis, oxidized prolactin, autoimmunity, malnutrition, genetic susceptibility, and apoptosis. PPCM is still a diagnosis of exclusion. Biomarkers with a strictly pregnancy-related kinetics are unavailable in clinical practice, and their role has not yet been fully elucidated. Globally, prognosis has slightly improved over the past years. In some patients, the clinical status quickly improves and sometimes returns to normal. In others, clinical conditions rapidly worsen and are unresponsive to medical therapy, and chronic heart failure (HF) from persistent left ventricular dysfunction (LVD) develops. Acute care treatment may involve the use of intravenous vasodilators, inotropes, with a prominent role for levosimendan, intra-aortic balloon pump, ventricular assist devices, and heart transplant. Beta-1-adrenergic agonists may promote myocyte loss and HF in PPCM patients, induce permanent damage, and prevent recovery. Novel disease-specific therapeutic concepts, such as targeting $16 \mathrm{kDa}$ prolactin and its downstream mediator miR-146a and/or the vascular endothelial growth factor (VEGF) system, may promote healing. Bromocriptine, a dopamine 2D agonist which blocks prolactin, may be a disease-specific treatment for PPCM in addition to standard therapy in patients with acute onset of the disease. Survivors of PPCM often recover from LVD; however, they may be at risk for HF recurrence and death in subsequent pregnancies. Women with chronic LVD should be managed according to international guidelines, and subsequent gestations should be avoided. Close follow-up and accurate risk stratification are mandatory for women who have recovered from PPCM and need counseling for future pregnancies.
\end{abstract}

Keywords: peripartum cardiomyopathy, postpartum cardiomyopathy, prolactin, microRNA, biomarker, acute heart failure, subsequent pregnancies

\section{Introduction}

Peripartum cardiomyopathy (PPCM) was recently redefined by the European Society of Cardiology (ESC) as an idiopathic cardiomyopathy presenting with heart failure (HF) due to systolic dysfunction of the left ventricle (left ventricular ejection fraction $[\mathrm{LVEF}]<45 \%$ ) toward the end of pregnancy or in the months following delivery, where no other cause of HF is found. ${ }^{1}$ The previous definition "development of HF during the last month of pregnancy or within months after delivery", that encompassed a strict time limit to the diagnosis, was eliminated.
Correspondence: Angela Beatrice Scardovi

Division of Cardiology, S. Spirito

Hospital, Lungotevere in Saxia I, 00193

Rome, Italy

Tel +393388479212

Fax +39668352458

Email ab.scardovi @libero.it 
PPCM and pregnancy-associated cardiomyopathy are part of the same clinical spectrum, and some patients may present with PPCM symptoms earlier than the last gestational month. Until specific etiologies are identified, PPCM remains a diagnosis of exclusion. ${ }^{1}$

Data from the international registry set up within the framework of the ESC EURObservational Research Program ${ }^{2}$ indicate that PPCM occurs worldwide, with remarkably similar clinical characteristics despite wide sociodemographic differences.

The disease is rare: depending on the geographical location, the reported incidence in the literature ranges from $1: 300$ up to $1: 15,000$ ( $<0.1 \%$ of pregnancies). Some studies suggest that the incidence of PPCM is one in 3,000 live births. However, one African study found it to be 1:1,000 live births.

In the US, administrative data suggest that the incidence of PPCM may have risen in the last decade, from 8.5 to 11.8 per 10,000 live births, with stable maternal adverse events rates. $^{3}$

There is also a very high risk of relapse in subsequent pregnancies, even following full recovery of left ventricular (LV) function after the first pregnancy. ${ }^{4-7}$

Morbidity and mortality rates are high, ranging from 5\% to $32 \% .{ }^{4,5}$ The prognosis is dependent on recovery from $\mathrm{HF}$ during the first 6 months postpartum with approximately $28 \%$ lethality.

Because the symptoms of PPCM overlap with those of idiopathic cardiomyopathy, definite diagnosis and subsequent management of PPCM require a high index of suspicion. Many women who initially present with signs and symptoms indicative of PPCM are diagnosed with "nonspecific symptoms of the puerperal period". Misdiagnosis of PPCM, which often leads to clinical deterioration and in some instances death, represents a target for early intervention and prevention.

Therefore, early and definitive diagnosis of PPCM is essential to limit the high risk of morbidity and mortality in both current and subsequent pregnancies.

There are a number of known risk factors, such as multiparity and age of the mother over 30 years, but the disease may appear in women without any risk factors. The diagnosis is mainly carried out using echocardiography which shows a clear reduction of systolic LV function. The therapeutic approach is generally the same as for idiopathic cardiomyopathy, but caution should be exercised for pregnancy-related drug contraindications. In the peripartum phase, a close cooperation between the disciplines of cardiology, cardiac surgery, neonatology, obstetrics, and anesthesiology seems to be the upcoming winning strategy.

\section{Etiopathogenesis}

The precise mechanisms that lead to PPCM remain poorly defined. Many pathogenetic processes have been suggested: viral myocarditis, an abnormal immune response to fetal micro-chimerism (harboring of fetal cells in maternal circulation), a maladaptive response to the hemodynamic stresses of pregnancy, stress-activated cytokines, and prolonged tocolysis. ${ }^{1,8-12}$

During pregnancy, blood volume and cardiac output increase. ${ }^{12,13}$ In addition, afterload decreases because of vascular smooth muscle relaxation. ${ }^{14}$ These changes cause a transient, and reversible, hypertrophy of the left ventricle to meet the needs of the mother and fetus. ${ }^{15}$ The transient attending diastolic dysfunction during the third trimester and early postpartum period resolves shortly after birth in a normal pregnancy. ${ }^{1,15}$ Pearson et al suggested that PPCM might be due, in part, to an exaggerated decrease in LV function when these hemodynamic changes of pregnancy occur. ${ }^{13}$ In addition, nutritional disorders, such as deficiencies in selenium and other micronutrients, might play a role in the pathogenesis of PPCM. ${ }^{12,16}$ Also, a familial predisposition to PPCM has been reported. ${ }^{17-20}$

Although underlying genetic variants common to dilated cardiomyopathies are being proposed, a genetic basis specific to PPCM has not been systematically studied. ${ }^{19}$

van Spaendonck-Zwarts et al described a cohort of 18 families who showed a high proportion of titin mutations and a low recovery rate in PPCM cases. ${ }^{20}$

The ESC currently classifies PPCM as a nonfamilial, nongenetic form of dilated cardiomyopathy. ${ }^{1}$

The upcoming pathophysiological hypothesis consists in a "two-hit" model of angiogenic imbalance in the heart during the peripartal period, whereby systemic antiangiogenic signals during late pregnancy act on a host susceptibility milieu with insufficient local proangiogenic defenses in the heart. ${ }^{4,5,21}$ The higher rates of hypertensive disorders and preeclampsia (22\% vs 3-5\%) found in PPCM patients than observed during pregnancy further support the hypothesis of a common vascular pathobiology. ${ }^{22,23}$

Recent findings in human disease support this notion. Damp et al assessed the time course and relation with LV function of relaxin-2, prolactin, and sFlt1, an antiangiogenic factor released from the placenta peripartum, in 98 women presenting postdelivery with PPCM. They found higher levels of relaxin-2 in women with less remodeling and more frequent recovery; conversely, worse symptoms and prognosis were associated with higher sFlt1 concentrations. ${ }^{24}$ Since VEGF, which is inhibited by sFlt1, partly mediates 
the vasodilatory and proangiogenic effects of relaxin-2, the findings observed by Damp et al in humans support the notion of an imbalance in angiogenesis as a driver of PPCM instauration and clinical outcomes. Angiogenic imbalance may persist even after recovery. In a cohort of 29 PPCM patients with LV function recovery, Goland et $\mathrm{al}^{23}$ observed higher concentrations of soluble VEGF receptor sFlt1 and decreased levels of circulating endothelial progenitor cells and VEGF than in controls.

Angiogenic imbalance can further be triggered by oxidative stress-mediated activation of the protease cathepsin D, a protease responsible for the cleavage of the angiostatic and proapoptotic $16-\mathrm{kDa}$ subfragment from the nursing hormone prolactin. $^{25}$

An increased concentration of plasma inflammatory cytokines, specifically tumor necrosis factor and Fas/Apo-1, which plays a key role in apoptosis (programmed cell death), has been identified in women with PPCM as compared to healthy volunteers and among PPCM non-survivors compared to survivors. ${ }^{26}$

Hilfiker-Kleiner et al proposed a key role for prolactin in the pathogenetic mechanism of PPCM. ${ }^{27}$ Prolactin levels are associated with an increased blood volume, a decreased blood pressure, a decreased angiotensin responsiveness, and a reduction in the levels of water, sodium, and potassium. Prolactin also increases the concentrations of circulating erythropoietin, and hence hematocrit levels. ${ }^{12}$ Mice bred to have a cardiomyocyte-specific deletion of STAT3, a protein that plays a key role in many cellular processes such as cell growth and apoptosis, develop PPCM. ${ }^{28}$ STAT3 deletion blunts the induction of the antioxidant enzyme manganese superoxide dismutase, and the resulting increased oxidative stress leads to enhanced expression of cardiac cathepsin D that promotes cleavage of the $23-\mathrm{kDa}$ prolactin (PRL) into an angiostatic and proapoptotic $\mathrm{N}$-terminal $16-\mathrm{kDa}$ fragment (16-kDa PRL). The 16-kDa PRL causes massive endothelial damage and myocardial hypoxemia and dysfunction. In partial support of animal data, low STAT3 protein levels were found by Hilfiker-Kleiner et al in myocardial tissue from five women with PPCM who underwent heart transplant. These authors also detected an antigen like $16-\mathrm{kDa}$ prolactin in sera from three nursing PPCM patients but not in healthy controls. ${ }^{28}$

Moreover, in STAT3-deficient mice, the postpartum enhancement of PI3K/Akt signaling, a pathway that is highly activated by increased mechanical stress and high estrogen levels and promotes cardiac adaptation with physiological hypertrophy during pregnancy, was found to be detrimental and to accelerate cardiac inflammation and fibrosis. ${ }^{29}$ The development of fibrosis and inflammation was attenuated, and PPCM lethality was also prevented by bromocriptine in this model.

Hence, prolactin and its cleaved $16-\mathrm{kDa}$ form seem to be pivotal for PPCM as suggested by the protection from the disease by prolactin blockade. However, although promising and fascinating, this theory remains experimental and insufficiently proven in humans, while increased serum levels of 16-kDa PRL or cathepsin D in PPCM patients have not been consistently documented till now.

\section{Diagnosis}

The onset of PPCM can easily be masked - and missedbecause the manifestations can mimic those of mild HF. Women with PPCM most commonly show dyspnea, dizziness, chest pain, cough, neck vein distension, fatigue, and peripheral edema. ${ }^{1}$ Arrhythmias, embolic events from thrombosis of the dilated, dysfunctional left ventricle, and acute myocardial infarction due to decreased perfusion to the coronary arteries are other, less common clinical features. ${ }^{1,5,10-12}$

Typical HF signs include hypoxia, jugular venous distention, S3 and S4 gallop, rales, and hepatomegaly. ${ }^{12,18}$ Blood pressure is often normal or decreased, and tachycardia is common. ${ }^{12}$

The electrocardiogram (ECG) appears to be a useful firstline tool in both screening and prognostication in resourcepoor settings such as Africa. ${ }^{30}$

An ECG should be performed in all patients with suspected PPCM as it has a high negative predictive value. Indeed, despite the absence of a specific ECG pattern for PPCM, at initial evaluation, the ECG is rarely normal: repolarization abnormalities, LV hypertrophy, dysrhythmias, Q-waves in the anteroseptal precordial leads, and prolonged PR and QRS intervals are common. ${ }^{12,31}$

Measurement of natriuretic peptides may help during screening for identifying a cardiac origin of dyspnea, although it does not differentiate PPCM from other cardiomyopathies. ${ }^{1,11}$ More specific biomarkers would be helpful to allow a faster and more reliable diagnosis of PPCM, but currently none have yet been defined. ${ }^{1}$ Serum level of troponin may be helpful in ruling out myocardial infarction; however, an increase in troponin in the acute phase of PPCM, without myocardial infarction, can occur. ${ }^{12}$

Several other laboratory tests should be performed: complete blood cell count and serum levels of troponin, urea, creatinine, and electrolytes, ${ }^{12}$ and liver and thyroid function tests.

The definitive diagnosis of PPCM depends on echocardiographic identification of new-onset HF during a limited period around parturition. 
A diagnosis of PPCM requires the exclusion of other causes of HF: myocardial infarction, sepsis, severe preeclampsia, pulmonary embolism, valvular diseases, and other forms of cardiomyopathy (Figure 1). 1,5,12,25

Chest radiographs may be helpful in acute pulmonary edema, but much less so if no clinical evidence of pulmonary congestion is revealed. On the other hand, congestion could be better identified by pulmonary echography. ${ }^{32}$

Cardiac magnetic resonance imaging has been suggested as a complementary tool in the diagnosis and evaluation of women with PPCM, to measure global and segmental myocardial contraction and to identify inflammatory processes. ${ }^{1,5,13,25}$ However, administration of gadolinium to assess late enhancement should be avoided until after delivery, unless necessary.

Endomyocardial biopsy does not add any diagnostic or prognostic information in the case of PPCM but can be used to rule out acute myocarditis after delivery. Only a few PPCM cases have been related to myocarditis so far, but myocarditis may underlie dilated cardiomyopathies and acute HF that can occur or worsen during pregnancy, and specific immunosuppressive or immune-modulatory treatments may be helpful for some forms of myocarditis. ${ }^{33,34}$

\section{Management}

Management of PPCM, in the absence of evidence-based data, is like standard treatment for other forms of HF. ${ }^{1,12,25,35}$
Timely diagnosis and delivery are crucial. Careful attention should be paid to fetal safety and to excretion of drugs or drug metabolites during breastfeeding after delivery. ${ }^{1,12,24}$ The goals of HF treatment are to improve hemodynamic status, minimize signs and symptoms, and optimize long-term outcomes. Treatment focuses on reducing preload and afterload and increasing cardiac inotropy. ${ }^{1,12,25,35}$

Patients who present after delivery should be treated according to international HF guidelines. ${ }^{35}$ For patients presenting during pregnancy, angiotensin-converting enzyme (ACE) inhibitors, angiotensin-receptor blockers, and renin inhibitors are contraindicated because of fetal toxicity. Hydralazine and nitrates can be used instead. After delivery, ACE inhibitors can be started, but during breastfeeding, captopril or enalapril should be preferred. Despite an increased risk of fetal growth restriction, beta-blockers are indicated in all stable patients, with metoprolol succinate being the preferred agent. ${ }^{12}$ Mineralocorticoid receptor antagonists (MRAs) should be avoided during pregnancy and lactation, but should be started afterwards in stable patients. Diuretics should be administered with caution during pregnancy as they may impair placental perfusion. ${ }^{12}$

ACE inhibitors, beta-blockers, and MRAs should be continued at least till after complete recovery of LV size and systolic function. Discontinuation of HF medications can be considered only in the case of complete recovery of ventricular function and exercise response.

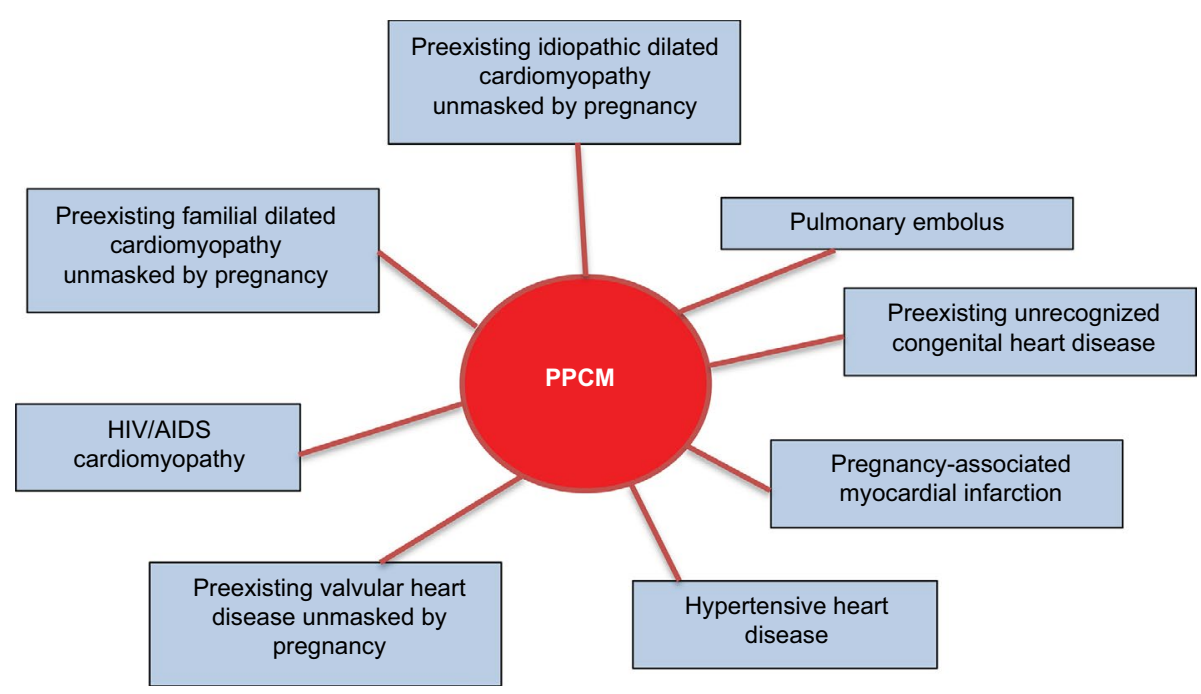

Figure I Differential diagnosis of PPCM.

Note: The diagnosis of PPCM requires careful exclusion of other common causes of ventricular dysfunction and heart failure in pregnancy.

Abbreviation: PPCM, peripartum cardiomyopathy. 
Initial treatment with ivabradine, aimed at controlling tachycardia even before or in parallel with beta-blockers, appears to be safe and effective. ${ }^{1}$

An interdisciplinary approach with the involvement of cardiologists, intensivists, obstetricians, neonatologists, anesthetists, and cardiac surgeons is needed in cases of severe acute HF (Figure 2). The initial treatment of patients with severe forms of acute PPCM is significantly different from the one for stable patients, and rapid access to intensive care is mandatory. ${ }^{1}$ Intravenous administration of positive inotropic agents is required to restore hemodynamics in the case of cardiogenic shock, and optimization of oxygenation is one of the main targets. Noninvasive ventilation reduces respiratory distress and may decrease intubation and mortality rates. ${ }^{1}$ Intubation with mechanical ventilation should be considered in the case of altered mental state or persistent hypoxemia.

Dobutamine and milrinone are not contraindicated in pregnancy. ${ }^{1}$ Milrinone has vasodilating properties for both the systemic and the pulmonary circulation, a mechanism that may provide additional benefit over other inotropic agents such as catecholamines that are associated with adverse effects in patients with advanced HF. ${ }^{1,12}$ Dobutamine is less favorable in PPCM patients due to metabolic compromise and it requires beta-receptors for its inotropic effects. ${ }^{12}$ Therefore, catecholamines should be avoided whenever possible or used only with extreme caution.

Levosimendan, in contrast to dobutamine and adrenaline, does not increase myocardial oxygen demand and may be considered as the upcoming inotropic agent for patients with severe PPCM. ${ }^{36}$ Although a prospectively randomized trial showed that the addition of levosimendan to conventional therapy did not improve the outcome in patients with PPCM, a recent study found that the use of levosimendan in patients with PPCM induced rapid hemodynamic recovery. ${ }^{1,37,38}$ In any case, adrenaline should be avoided, while noradrenaline should be the first-line vasopressor whenever required by the clinical condition.

Vasodilators such as nitroglycerin and nitroprusside also may be of benefit. ${ }^{12}$ Nitroprusside should be used with caution in pregnant women because the toxic effects of thiocyanate can be harmful to the fetus. ${ }^{12}$

Prolactin blockade has shown promising results in several case series and in a small proof-of-concept trial: ${ }^{39}$ bromocriptine, at a starting dose of $2.5 \mathrm{mg}$ twice daily, seems to be able

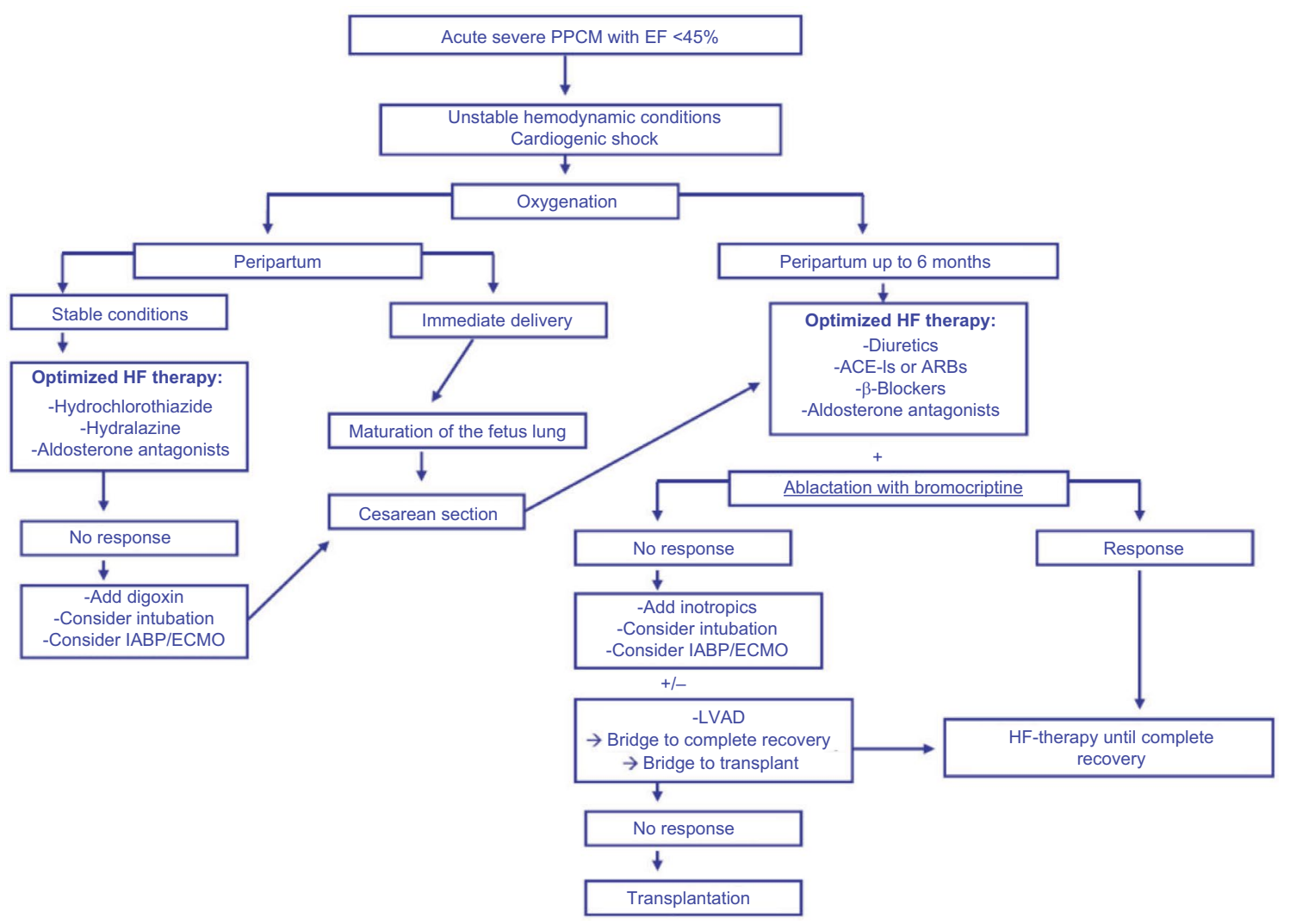

Figure 2 Therapeutic algorithm for acute severe presentations of PPCM.

Abbreviations: PPCM, peripartum cardiomyopathy; EF, ejection fraction; HF, heart failure; IABP, intra-aortic balloon pump; ECMO, extracorporeal membrane oxygenation; ACE-Is, angiotensin-converting enzyme inhibitors; ARBs, angiotensin II receptor blockers; LVAD, left ventricular assist device. 
to prevent the deterioration in LV size and systolic function when given in addition to standard HF therapy ${ }^{39}$ and should be considered for patients with cardiopulmonary distress. Other small studies and case reports suggested that cabergoline, another potent dopamine receptor antagonist like bromocriptine, could be beneficial. ${ }^{40,41}$ Because of the long half-life of cabergoline, 14-21 days, a single dose is often enough. To prevent thromboembolic events during the use of bromocriptine, treatment should always be accompanied by at least prophylactic anticoagulation with heparin. ${ }^{42}$

The combination of reduced EF and the procoagulant activity during the peripartal phase exposes patients to a clinically important risk of cardioembolic events. In the ESC registry of worldwide PPCM, thromboembolic events were reported in $6.8 \%$ of cases. ${ }^{2}$ Anticoagulation with heparin should also be started in all patients with acute PPCM and severely reduced LV systolic function ( $\mathrm{LVEF} \leq 35 \%$ ).

Arrhythmias should be aggressively treated to minimize thrombus formation and to optimize cardiac function. ${ }^{12,13}$

Immunosuppressive and anti-inflammatory therapies have not improved the outcome in PPCM, and in general, are not recommended. ${ }^{43}$

Medical therapy can be unsuccessful in women with PPCM, and mechanical cardiovascular support with an intra-aortic balloon pump (IABP) or ventricular assist devices may be required as a "bridge to recovery" or to transplantation. 1,12,13,44

If needed, a device for temporary support should be implanted in the acute phase, either as "bridge to recovery", if ventricular function improves during the subsequent days and weaning can be achieved, or as "bridge to bridge", if hemodynamic impairment persists and circulatory support must be ensured. Because of the higher proportion of patients with at least partial recovery of ventricular function compared with other cardiomyopathies, an initial "bridgeto-transplantation" strategy is seldom necessary. First-line devices can be chosen among IABP, ImpellaR, or surgical equipment (e.g. CentriMagR, AbiomedBVS 5000R, TandemHeartR, veno-arterial extracorporeal membrane oxygenation) considering the local availability and the experience of the involved care team. Evaluation of right ventricular function is crucial for the strategy. In the presence of impaired right ventricular function, a biventricular assist device (BiVAD or total artificial heart) may be chosen (e.g. Berlin Heart EXCORR). ${ }^{1,12,13,44}$

Women in whom maximal medical management is unsuccessful may be candidates for cardiac transplantation. Cardiac transplantation could be necessary in about $4 \%$ of women with PPCM unresponsive to medical therapies. ${ }^{12}$
Previous small series reported comparable outcomes for PPCM recipients to other transplanted patients. Conversely, recent findings from the United Network for Organ Sharing Database, ${ }^{45}$ on 485 PPCM recipients, that represented $1 \%$ of all transplants, and 5\% of female transplants, showed worse posttransplant outcomes, with higher rejection rates within the first year, lower graft and age-adjusted survival, in PPCM patients than in other women and all other recipients in this large registry. Since PPCM patients, though younger, showed higher sensitization rates than other women, the authors concluded that factors challenging prognosis in these women should be actively sought, rejection should be treated more aggressively, and optimal compliance should be obtained.

\section{Prevention of sudden cardiac death}

It is assumed that about a quarter of deaths are caused by ventricular tachyarrhythmia, mostly occurring during the first 6 months, mainly in patients with severely impaired cardiac function. ${ }^{46}$ Current ESC guidelines for the treatment of HF recommend implantation of an implantable cardioverter defibrillator (ICD) for primary prevention in patients with symptomatic $\mathrm{HF}$ and $\mathrm{LVEF} \leq 35 \%$ despite optimal pharmacological treatment or for secondary prevention in patients with documented ventricular arrhythmia causing hemodynamic instability. ${ }^{35}$

In the context of PPCM, the potential for complete recovery of ventricular function should be considered, and decisions about implantation of an ICD may be very challenging, considering the uncertainty about the subsequent evolution of ventricular function and the young age of the patients. Indeed, several publications reported recovery of LV function in at least $50 \%$ of subjects within 6 months after diagnosis. ${ }^{46,47}$ However, a Turkish study reported delayed recovery (after 6 months) in a sizable proportion of patients. ${ }^{48}$ A recent retrospective study from the US showed complete recovery of LV function in $23 \%$ of patients and partial recovery in another $19 \%$ over a mean duration of $33 \pm 21$ months, confirming that delayed recovery over 6 months is frequent (83\%). ${ }^{49}$ In this study, African-American women showed lower rates of recovery compared with Caucasians, and postpartum presentation was a predictor of good recovery.

Considering these observations, early implantation of an ICD in patients with newly diagnosed PPCM is not appropriate. On the other hand, postponement of ICD implantation beyond 6-12 months exposes the patients to an unacceptable risk of sudden cardiac death.

Wearable cardioverter defibrillator (LifeVestR; Zoll, Pittsburgh, PA, USA) offers, for at least 3-6 months, an interesting 
compromise for the prevention of sudden cardiac death in the first period after diagnosis, until a definitive decision about ICD implantation can be made. ${ }^{35,49}$ In patients who do not recover despite 3-6 months on optimized HF therapy, a conventional recommendation for the primary prophylactic implantation of an ICD applies. ${ }^{35,50}$ Subcutaneous ICDs, avoiding intravascular leads and thus potential infectious complication, represent an alternative to transvenous systems.

Although no specific data on device therapy in patients with PPCM exist, in patients with $\mathrm{HF}, \mathrm{LVEF} \leq 35 \%$ despite optimal medical therapy for at least 3-6 months, and left bundle branch block, cardiac resynchronization theraphy (CRT) is indicated, according to the current ESC guidelines on CRT following recommendations for patients with dilated cardiomyopathy. ${ }^{35}$

\section{Prognosis}

Despite increasing knowledge about the epidemiology and pathophysiology of PPCM, mortality rates are not well described and vary widely, ranging from $<5 \%$ up to $50 \%$. ${ }^{1,7,12,47}$ Earlier diagnosis, coupled with modern management of HF, has a pivotal role in influencing the mortality associated with PPCM. ${ }^{7,12,47}$

The prognosis in PPCM varies geographically. In the US, reported mortality rates range between $0 \%$ and $19 \%$, while rates of cardiac transplantation vary from $6 \%$ to $11 \%{ }^{51}$ Substantial differences in the reports are probably due to variations in patient populations, diagnostic criteria, and treatments, as well as reporting bias. ${ }^{51}$ Mortality was found to be higher when baseline LVEF was $\leq 25 \%$, as well as in women in whom the diagnosis of PPCM was delayed. ${ }^{52}$

In addition, mortality was reported to increase with maternal age, in women with parity of $>4$, and in African-American women, who were 6.4 times more likely to die compared with White women. ${ }^{53}$ Eighteen percent of deaths occurred within 1 week, and $87 \%$ within 6 months of diagnosis, due to either progressive HF or sudden cardiac death. In South Africa, a case series has demonstrated that mortality slowly improved over time, but 6-month and 2-year death rates remained at $10 \%$ and $28 \%$, respectively. ${ }^{25}$

Overall, LV systolic function returned to normal in about $23-41 \%$ in a case series in the US, Haiti, and Turkey. ${ }^{25}$

In a German registry of 115 prospectively enrolled patients with confirmed PPCM, 16.5\% had a positive family history of cardiomyopathy, and mean LVEF was $27 \%$. In this study, higher serum levels of cathepsin D, the enzyme that generates $16-\mathrm{kDa}$ prolactin, and miR-146a, a direct target of $16-\mathrm{kDa}$ prolactin, were found in PPCM patients than in healthy matched controls studied postdelivery. ${ }^{54}$ These biomarkers support the pathophysiological role of $16-\mathrm{kDa}$ PRL in PPCM and may become useful, after eventual validation, for specific diagnostic profiling.

In the German registry, after an average 6-month followup, $85 \%$ of patients showed LVEF improvement and $47 \%$ full recovery, while $15 \%$ failed to recover and $2 \%$ died. ${ }^{53}$ Hypertension developing during pregnancy was associated with a better prognosis, while a baseline LVEF $\leq 25 \%$ purported a worse outcome. A high recovery rate (96\%) was observed in patients on combination therapy with betablockers, ACE inhibitors, or angiotensin receptor blockers, and bromocriptine.

Similar outcome rates were found in the contemporary NHLBI multicenter Investigations of Pregnancy-Associated Cardiomyopathy (IPAC) study. ${ }^{55}$ Out of 100 PPCM patients enrolled in IPAC under a background of optimal medical treatment including renin-angiotensin system antagonists and beta-blockers, but a very low rate of PRL blockade ( $1 \%$ ), only $13 \%$ developed major events or persistent systolic dysfunction, while $72 \%$ showed full recovery of ventricular function. ${ }^{55}$

Moreover, the IMAC2 investigators demonstrated a definitely higher recovery rate in a comparison of PPCM patients with other women and men affected by recent-onset cardiomyopathy of a different origin (48\%, 34\% and 19\%, respectively) and a higher LV function level after adjustment for age and baseline $\mathrm{LVEF}^{56}$

Hence, the current outlook with contemporary HF drug treatment seems more favorable than previously reported.

Independent predictors of LV function recovery in IPAC were LV dimensions at diagnosis, and race. The IPAC study was moreover the first study to establish a relationship between genetic background and differences in LV remodeling and recovery in women with PPCM. ${ }^{57}$ Black women are more predisposed to PPCM and less likely to recover. The TT genotype in the GNB3 C825T, which is associated with hypertension and with African-American descent, was associated with lower LVEF at 6 and 12 months postpartum. Women presenting with PPCM with this specific genotype may be at higher risk for chronic cardiomyopathy.

\section{Subsequent pregnancies}

For women who survive PPCM, a thorny issue is whether they can safely become pregnant again. ${ }^{12,58}$ Because established recommendations for future pregnancies do not exist, joint cardiologic and obstetric management including counseling on the potential risk of PPCM recurrence with future pregnancies is recommended. ${ }^{18}$ 
Relapse with symptom worsening and deterioration of LV function occurs in almost one-third of the cases. ${ }^{58}$ The risk of relapse and mortality is substantially higher in patients with persistent LV dysfunction; hence, LV recovery is considered the most reliable prognostic predictor of survival in subsequent pregnancies, ${ }^{56}$ but it does not guarantee an uncomplicated subsequent pregnancy. ${ }^{18}$ Mechanisms underlying recurrence risk are presently unknown. ${ }^{59}$ Although mortality in such patients is rare, marked decreases in LV function have been reported in approximately $20 \%$ of patients, with persisting dysfunction after pregnancy in about $50 \%{ }^{25,58}$

A multicenter study from Germany, South Africa, and Scotland ${ }^{60}$ reported outcomes with subsequent pregnancies in 30 PPCM patients. Half of them had not fully recovered LV function before the subsequent pregnancy: four deaths occurred and these patients showed lower cardiac function early postdelivery and a lower rate of full recovery during follow-up. Moreover, even among patients who had fully recovered cardiac function before the subsequent pregnancy, sustained normal function was only observed in 56\%. Hence, even PPCM patients with fully recovered LV function still have a substantial risk for relapse. The authors reported a higher LVEF and full recovery rate in the women (70\%) who had received bromocriptine in addition to standard HF therapy.

Women with persistent HF should be discouraged from subsequent pregnancies; the risks are 19\% higher for maternal death than among women with PPCM whose HF has resolved because the heart cannot tolerate the increased cardiovascular workload. ${ }^{12,58}$

Women whose cardiomyopathy appears to have resolved are an even more difficult group to counsel, since the risk of PPCM recurrence may persist. ${ }^{12,58}$ One in five women with apparent full recovery are at risk of moderate-to-severe deterioration of LV function during a subsequent pregnancy, which is sustained after delivery in $20-50 \%$ of cases. Moreover, PPCM has been associated with multiparity, and despite limited information available, subsequent pregnancies can increase the risk for recurrent episodes of PPCM, irreversible cardiac damage, decreased LV function, and even death. ${ }^{1,11,58,60}$

Classification of PPCM patients into two categories (recovered vs non-recovered LV function) has been suggested as the most appropriate counseling strategy for future pregnancy. ${ }^{29}$ However though cardiac function is normalized in the former group, LV contractile reserve may still be impaired, and recurrence of PPCM is possible. ${ }^{12,58,60}$ Sustained recovery of LVEF after withdrawal of HF drugs is another favorable indicator of no recurrence. ${ }^{40,58}$
There is limited knowledge on fetal safety in PPCM patients who undergo a subsequent pregnancy. Higher rates of abortion and premature delivery have been observed in patients with LV dysfunction than in those with LV function recovery, in some series, ${ }^{58}$ but not in others. ${ }^{60}$

In any case, intensive monitoring during pregnancy and after delivery is mandatory to detect early recurrence and promptly instate HF treatment.

\section{Future directions and conclusion}

PPCM appears to be primarily an endothelial disease, and variable cardiac phenotype suggests information on etiology and pathophysiology. In some subsets of patients, hypertension during pregnancy is the prominent risk factor and may predispose to PPCM by initiating a powerful antiangiogenic environment. Different phenotypes and biomarkers underlying the spectrum between preeclampsia, with prominent diastolic impairment, and full-blown PPCM with impaired systolic function should be investigated to establish the mechanism of their interaction to precipitate cardiac failure.

The ability to predict recovery of LV function after a patient develops PPCM remains a significant challenge to clinicians. Among the many predictors examined, including clinical characteristics, biohumoral factors, and echocardiographic features, vascular homeostasis and genetic profiling promise to further our understanding of the physiology underlying the development of PPCM. Patient genotype may help clinicians to better individualize discussions with patients regarding the ramifications of the cardiomyopathy, and help potentially tailor medical therapy individually to women with PPCM.

The role of biomarkers outside the experimental setting has not yet been fully elucidated and should represent a field of active research. Serum levels of natriuretic peptides, C-reactive protein, and miR-146a may be helpful for early diagnosis and risk stratification, but are not specific to PPCM. The preliminary findings on the opposite associations of elevated relaxin- 2 with early functional recovery and elevated sFlt1 with adverse prognosis are attuned to the hypothesis of angiogenic imbalance and highlight the potential role of biomarkers as risk stratification tools and future therapeutic targets in PPCM. However, these biomarkers with a strictly pregnancy-related kinetics are not available in clinical practice, while other proposed disease-specific humoral indicators ${ }^{4}$ still await validation.

The rising incidence of the disease may be related to greater awareness or socioeconomic or risk factor changes. Globally, the prognosis has slightly improved recently. In 
acute care, levosimendan may represent the drug of choice as opposed to beta-1-adrenergic agonists which, by promoting cardiac damage, may hinder recovery.

Careful assessment of risk factors in pregnant women could help prevent PPCM. Novel disease-specific therapeutic concepts, such as targeting 16-kDa prolactin and its downstream mediator miR-146a and/or the VEGF system, might represent the upcoming option for therapy or prevention in patients at risk, with the caution that current results are not clinically robust and large appropriately designed clinical trials are needed.

Women with chronic LV dysfunction should be managed according to international guidelines, and subsequent gestations should be avoided. Close follow-up and accurate risk stratification are mandatory for women who have recovered from PPCM and need counseling for future pregnancies.

\section{Disclosure}

The authors report no conflicts of interest in this work.

\section{References}

1. Bauersachs J, Arrigo M, Hilfiker-Kleiner D, et al. Current management of patients with severe acute peripartum cardiomyopathy: practical guidance from the Heart Failure Association of the European Society of Cardiology Study Group on peripartum cardiomyopathy. Eur J Heart Fail. 2016;18(9):1096-1105.

2. Sliwa K, Mebazaa A, Hilfiker-Kleiner D, et al. Clinical characteristics of patients from the worldwide registry on peripartum cardiomyopathy (PPCM): EURObservational Research Programme in conjunction with the Heart Failure Association of the European Society of Cardiology Study Group on PPCM. Eur J Heart Fail. Epub 2017 Mar 8.

3. Kolte D, Khera S, Aronow WS, et al. Temporal trends in incidence and outcomes of peripartum cardiomyopathy in the United States: a nationwide population-based study. J Am Heart Assoc. 2014;3(3):e001056.

4. Hilfiker-Kleiner D, Sliwa K. Pathophysiology and epidemiology of peripartum cardiomyopathy. Nat Rev Cardiol. 2014;11(6):364-370.

5. Hilfiker-Kleiner D, Haghikia A, Nonhoff J, Bauersachs J. Peripartum cardiomyopathy: current management and future perspectives. Eur Heart J. 2015;36(18):1090-1097.

6. Fett JD, Christie LG, Carraway RD, Murphy JG. Five-year prospective study of the incidence and prognosis of peripartum cardiomyopathy at a single institution. Mayo Clin Proc. 2005;80(12):1602-1606.

7. Mielniczuk LM, Williams K, Davis DR, et al. Frequency of peripartum cardiomyopathy. Am J Cardiol. 2006;97(12):1765-1768.

8. Felker GM, Jaeger CJ, Klodas E, et al. Myocarditis and long-term survival in peripartum cardiomyopathy. Am Heart J. 2000;140(5):785-791.

9. Ansari AA, Fett JD, Carraway RE, Mayne AE, Onlamoon N, Sundstrom JB. Autoimmune mechanisms as the basis for human peripartum cardiomyopathy. Clin Rev Allergy Immunol. 2002;23(3):301-324.

10. Abboud J, Murad Y, Chen-Scarabelli C, Saravolatz L, Scarabelli TM. Peripartum cardiomyopathy: a comprehensive review. Int J Cardiol. 2007;118(3):295-303.

11. Ntusi NB, Mayosi BM. Aetiology and risk factors of peripartum cardiomyopathy: a systematic review. Int J Cardiol. 2009;131(2):168-179.

12. Johnson-Coyle L, Jensen L, Sobey A; American College of Cardiology Foundation; American Heart Association. Peripartum cardiomyopathy: review and practice guidelines. Am J Crit Care. 2012;21(2):89-98.
13. Pearson GD, Veille JC, Rahimtoola S, et al. Peripartum cardiomyopathy: National Heart, Lung, and Blood Institute and Office of Rare Diseases (National Institutes of Health) workshop recommendations and review. JAMA. 2000;283(9):1183-1188.

14. Ramaraj R, Sorrell VL. Peripartum cardiomyopathy: causes, diagnosis, and treatment. Cleve Clin J Med. 2009;76(5):289-296.

15. Tidswell M. Peripartum cardiomyopathy. Crit Care Clin. 2004;20(4): 777-788.

16. Demakis JG, Rahimtoola SH, Sutton GC, et al. Natural course of peripartum cardiomyopathy. Circulation. 1971;44(6):1053-1061.

17. Pearl W. Familial occurrence of peripartum cardiomyopathy. Am Heart J. 1995;129(2):421-422.

18. Moioli M, Valenzano Menada M, Bentivoglio G, Ferrero S. Peripartum cardiomyopathy. Arch Gynecol Obstet. 2010;281(2):183-188.

19. van Spaendonck-Zwarts KJ, van Tintelen JP, van Veldhuisen DJ, et al. Peripartum cardiomyopathy as a part of familial dilated cardiomyopathy. Circulation. 2010;121(20):2169-2175.

20. van Spaendonck-Zwarts, KY, Posafalvi A, van den Berg MP, et al. Titin gene mutations are common in families with both peripartum cardiomyopathy and dilated cardiomyopathy. Eur Heart J. 2014; 35(32):2165-2173.

21. Patten IS, Rana S, Shahul S, et al. Cardiac angiogenic imbalance leads to peripartum cardiomyopathy. Nature. 2012;485(7398):333-338.

22. Bello N, Rendon IS, Arany Z. The relationship between pre-eclampsia and peripartum cardiomyopathy: a systematic review and meta-analysis. J Am Coll Cardiol. 2013;62(18):1715-1723.

23. Goland S, Weinstein JM, Zalik A, et al. Angiogenic imbalance and residual myocardial injury in recovered peripartum cardiomyopathy patients. Circ Heart Fail. 2016;9(11):e003349.

24. Damp J, Givertz MM, Semigran M, et al; IPAC Investigators. Relaxin-2 and soluble Flt1 levels in peripartum cardiomyopathy: results of the multicenter IPAC study. JACC Heart Fail. 2016;4(5):380-388.

25. Sliwa K, Hilfiker-Kleiner D, Petrie MC, et al; Heart Failure Association of the European Society of Cardiology Working Group on Peripartum Cardiomyopathy. Current state of knowledge on aetiology, diagnosis, management, and therapy of peripartum cardiomyopathy: a position statement from the Heart Failure Association of the European Society of Cardiology Working Group on peripartum cardiomyopathy. Eur J Heart Fail. 2010;12(8):767-778.

26. Sliwa K, Skudicky D, Bergemann A, Candy G, Puren A, Sareli P. Peripartum cardiomyopathy: analysis of clinical outcome, left ventricular function, plasma levels of cytokines and Fas/APO-1. J Am Coll Cardiol. 2000;35(3):701-705.

27. Hilfiker-Kleiner D, Sliwa K, Drexler H. Peripartum cardiomyopathy: recent insights in its pathophysiology. Trends Cardiovasc Med. 2008;18(5):173-179.

28. Hilfiker-Kleiner D, Kaminski K, Podewski E, et al. A cathepsin D-cleaved $16 \mathrm{kDa}$ form of prolactin mediates postpartum cardiomyopathy. Cell. 2007;128(3):589-600.

29. Ricke-Hoch M, Bultmann I, Stapel B, et al. Opposing roles of Akt and STAT3 in the protection of the maternal heart from peripartum stress. Cardiovasc Res. 2014;101(4):587-596.

30. Williams J, Mozurkewich E, Chilimigras J, Van De Ven C. Critical care in obstetrics: pregnancy-specific conditions. Best Pract Res Clin Obstet Gynaecol. 2008;22(5):825-846.

31. Tibazarwa K, Lee G, Mayosi B, Carrington M, Stewart S, Sliwa K. The 12-lead ECG in peripartum cardiomyopathy. Cardiovasc J Afr. 2012;23(6):322-329.

32. Platz E, Lewis EF, Uno $H$, et al. Detection and prognostic value of pulmonary congestion by lung ultrasound in ambulatory heart failure patients. Eur Heart J. 2016;37(15):1244-1251.

33. Fett JD. Peripartum cardiomyopathy may also present as "fulminant right ventricular myocarditis”. Am J Emerg Med. 2010;28(9):1056.

34. Maisch B, Ruppert V, Pankuweit S. Management of fulminant myocarditis: a diagnosis in search of its etiology but with therapeutic options. Curr Heart Fail Rep. 2014;11(2):166-177. 
35. Ponikowski P, Voors AA, Anker SD, et al; Authors/Task Force Members. 2016 ESC guidelines for the diagnosis and treatment of acute and chronic heart failure: the Task Force for the diagnosis and treatment of acute and chronic heart failure of the European Society of Cardiology (ESC). Developed with the special contribution of the Heart Failure Association (HFA) of the ESC. Eur Heart J. 2016;37(27): 2129-2200.

36. Arrigo M, Mebazaa A. Understanding the differences among inotropes. Intensive Care Med. 2015;41(5):912-915.

37. Biteker M, Duran NE, Kaya H, et al. Effect of levosimendan and predictors of recovery in patients with peripartum cardiomyopathy, a randomized clinical trial. Clin Res Cardiol. 2011;100(7):571-577.

38. Labbene I, Arrigo M, Tavares M, et al. Decongestive effects of levosimendan in cardiogenic shock induced by postpartum cardiomyopathy. Anaesth Crit Care Pain Med. 2017;36(1):39-42.

39. Sliwa K, Blauwet L, Tibazarwa K, et al. Evaluation of bromocriptine in the treatment of acute severe peripartum cardiomyopathy: a proofof-concept pilot study. Circulation. 2010;121(13):1465-1473.

40. Scardovi AB, De Maria R, Ricci R. Acute peripartum cardiomyopathy rapidly evolving in cardiogenic shock. Int J Cardiol. 2015;189: 255-256.

41. de Jong JS, Rietveld K, van Lochem LT, Bouma BJ. Rapid left ventricular recovery after cabergoline treatment in a patient with peripartum cardiomyopathy. Eur J Heart Fail. 2009;11(2):220-222.

42. Fett JD. Caution in the use of bromocriptine in peripartum cardiomyopathy. J Am Coll Cardiol. 2008;51(21):2083.

43. McNamara DM, Holubkov R, Starling, RC, et al. Controlled trial of intravenous immune globulins in recent-onset dilated cardiomyopathy. Circulation. 2001;103(18):2254-2259.

44. Gavaert S, Van Belleghem Y, Bouchez S, et al. Acute and critically ill peripartum cardiomyopathy and "bridge to" therapeutic options: a single center experience with intraaortic balloon pump, extra-corporeal membrane oxygenation and continuous-flow left ventricular assist devices. Crit Care. 2011;15(2):R93.

45. Rasmusson K, Brunisholz K, Budge D, et al. Peripartum cardiomyopathy: post-transplant outcomes from the United Network for Organ Sharing Database. J Heart Lung Transplant. 2012;31(2):180-186.

46. Duncker D, Haghikia A, König T, et al. Risk for ventricular fibrillation in peripartum cardiomyopathy with severely reduced left ventricular function: value of the wearable cardioverter/defibrillator. Eur J Heart Fail. 2014;16(12):1331-1336.

47. Brar SS, Khan SS, Sandhu GK, et al. Incidence, mortality, and racial differences in peripartum cardiomyopathy. Am J Cardiol. 2007; 100(2):302-304.
48. Biteker M, Ilhan E, Biteker G, Duman D, Bozkurt B. Delayed recovery in peripartum cardiomyopathy: an indication for long-term follow-up and sustained therapy. Eur J Heart Fail. 2012;14(8):895-901.

49. Pillarisetti J, Kondur A, Alani A, et al. Peripartum cardiomyopathy: predictors of recovery and current state of implantable cardioverterdefibrillator use. J Am Coll Cardiol. 2014;63(25 Pt A):2831-2839.

50. Brignole M, Auricchio A, Baron-Esquivias G, et al. 2013 ESC Guidelines on cardiac pacing and cardiac resynchronization therapy: the Task Force on cardiac pacing and resynchronization therapy of the European Society of Cardiology (ESC). Developed in collaboration with the European Heart Rhythm Association (EHRA). Eur Heart J. 2013;34(2):2281-2329.

51. Elkayam U. Clinical characteristics of peripartum cardiomyopathy in the United States: diagnosis, prognosis and management. J Am Coll Cardiol. 2011;58(7):659-670.

52. Goland S, Modi K, Bitar F, et al. Clinical profile and predictors of complications in peripartum cardiomyopathy. J Card Fail. 2009;15(8): 645-650.

53. Whitehead SJ, Berg CJ, Chang J. Pregnancy-related mortality due to cardiomyopathy: United States, 1991-1997. Obstet Gynecol. 2003; 102(6):1326-1331.

54. Haghikia A, Podewski E, Libhaber E, et al. Phenotyping and outcome on contemporary management in a German cohort of patients with peripartum cardiomyopathy. Basic Res Cardiol. 2013;108(4):366.

55. McNamara DM, Elkayam U, Alharethi R, et al; IPAC Investigators. Clinical outcomes for peripartum cardiomyopathy in North America: results of the IPAC Study (Investigations of Pregnancy-Associated Cardiomyopathy). J Am Coll Cardiol. 2015;66(8):905-914.

56. Cooper LT, Mather PJ, Alexis JD, et al; IMAC2 Investigators.. Myocardial recovery in peripartum cardiomyopathy: prospective comparison with recent onset cardiomyopathy in men and nonperipartum women. J Card Fail. 2012;18(1):28-33.

57. Sheppard R, Hsich E, Damp J, et al; IPAC Investigators. GNB3 C825T polymorphism and myocardial recovery in peripartum cardiomyopathy: results of the multicenter Investigations of Pregnancy-Associated Cardiomyopathy Study. Circ Heart Fail. 2016;9(3):e002683.

58. Elkayam U. Risk of subsequent pregnancy in women with a history of peripartum cardiomyopathy. JAm Coll Cardiol. 2014;64(15):1629-1636.

59. Fett JD, Shah TP, McNamara DM. Why do some recovered peripartum cardiomyopathy mothers experience heart failure with a subsequent pregnancy? Curr Treat Options Cardiovasc Med. 2015;17(1):354.

60. Hilfiker-Kleiner D, Haghikia A, Masuko D, et al. Outcome of subsequent pregnancies in patients with a history of peripartum cardiomyopathy. Eur J Heart Fail. Epub 2017 Mar 27.
Research Reports in Clinical Cardiology

\section{Publish your work in this journal}

Research Reports in Clinical Cardiology is an international, peerreviewed, open access journal publishing original research, reports, editorials, reviews and commentaries on all areas of cardiology in the clinic and laboratory. The manuscript management system is completely online and includes a very quick and fair peer-review system.

\section{Dovepress}

Visit http://www.dovepress.com/testimonials.php to read real quotes from published authors. 